\title{
NANOTECNOLOGÍA Y NANOMEDICINA: UN NUEVO HORIZONTE PARA EL DIAGNÓSTICO Y TRATAMIENTO MEDICO
}

\section{NANOTECHNOLOGY AND NANOMEDICINE: A NEW HORIZON FOR MEDICAL DIAGNOSTICS AND TREATMENT}

\author{
DE SILVA MN ${ }^{1}$
}

El diagnóstico precoz se considera una de las metas más importantes en medicina. Una vez que se dispone del diagnóstico médico completo, es preciso un tratamiento adecuado para el paciente. En oftalmología, la detección temprana de trastornos como la degeneración macular, la retinosis pigmentaria, el cáncer ocular y la retinitis por citomegalovirus (la causa más común de ceguera en los pacientes con SIDA) es muy importante para procurar el tratamiento no tóxico apropiado. Por tanto ¿cómo harán avanzar la nanotecnología y la nanomedicina el campo del diagnóstico y tratamiento de las enfermedades?

La nanotecnología es capaz de hacer avanzar la ciencia y la tecnología mediante la manipulación de propiedades y procesos a nivel de átomos y moléculas, abarcando desde $1 \mathrm{~nm}$ hasta $100 \mathrm{~nm}$. Hay un interés creciente por la nanotecnología en laboratorios de todo el mundo y su impacto no se limita sólo a la medicina. Podemos esperar ver muchos avances revolucionarios en energía, transporte, defensa y electrónica, con un impacto potencial de enorme importancia en la economía mundial global. Algunas de las áreas en la asistencia sanitaria y la medicina que se espera progresen gracias a la nanotecnología son el diagnóstico médico con exámenes a nivel minúsculo y el diagnóstico por imágenes con el uso de agentes dirigidos para obtener imágenes de forma precisa tales como partículas cuánticas funcionales. La tecnología para la liberación de fármacos se verá revolucionada con los sistemas de liberación por nanoingeniería, que controlarán de forma exacta la dosis y el tiempo de liberación de la droga con la ayuda de nanopartículas y nanodispositivos.

Las enfermedades pueden ser identificadas utilizando biomarcadores, que interactúan con las moléculas relacionadas con la enfermedad presentes en la sangre, en los fluidos corporales o en los tejidos. Actualmente, se utilizan para el diagnóstico métodos comunes tales como el análisis genético y el análisis proteómico. Hay una creciente necesidad de mejorar la tecnología y la instrumentación relacionada con dichos métodos para realizar diagnósticos con mayor velocidad, resolución y sensibilidad. Esto requerirá el avance del bio-análisis en nano-escala y puede conseguirse sólo con la miniaturización de la instrumentación. Los micro- y nano-dispositivos fluídicos han hecho avanzar el campo gracias a su habilidad para llevar a cabo análisis de alto rendimiento en formato chip (1). Estos dispositivos fluídicos son capaces de incrementar el número de muestras procesadas y reducir el tiempo requerido para realizar un análisis con una alta sensibilidad y resolución. Además, un instrumento crítico de reconocimiento molecular, el espectrómetro de masa, puede ser integrado en un sistema de separación basado en un chip microfluídico (2). Otro abordaje para el reconocimiento biológico molecular selectivo es el uso de micro/nano-vigas funcionales. Ciertas moléculas relacionadas con la enfermedad pueden ser reconocidas mediante la monitorización de la deflexión o cambio en la masa de la viga debido a la interacción entre la entropía configuracional y la energética intermolecular inducida por reacciones biomoleculares específicas en la

\footnotetext{
1 Instituto Oftalmológico Bascom Palmer. Universidad de Miami, Facultad de Medicina Leonard M. Millar, Centro de Investigación de la Visión McKnight, 1638 NW 10th Ave, Miami, FL 33136, EEUU.

E-mail: MDeSilva@med.miami.edu
} 
superficie de la viga funcional. Estas vigas funcionales proporcionan un medio de detectar fragmentos de virus, marcadores de enfermedad y células precancerosas.

Otra área interesante es el diagnóstico por imagen. En el pasado, la bio-imagen utilizaba sustancias fluoradas, que tienen ciertas desventajas como la auto-fluorescencia, el hecho de que se apagan y la fijación no específica. La nanotecnología ha ayudado a desarrollar las partículas cuánticas (ejemplos: los nanocristales semiconductores con un corazón hecho de cadmio selénico o cadmio telúrico), que son capaces de regular las longitudes de ondas de absorción y emisión de acuerdo con el tamaño de la partícula. Se utiliza un armazón aislante para proteger el material semiconductor del núcleo de los grupos funcionales de la superficie, que reconocerán las moléculas relacionadas con la enfermedad. Se considera esto una atractiva aproximación para observar patología tisular y puede ser utilizada para aplicaciones quirúrgicas para identificar tejido en diferentes estadios de la enfermedad (4).

Habiendo ya abordado el tema del diagnóstico médico, vamos ahora a mirar cómo la nanotecnología puede jugar un papel en el tratamiento. La nanotecnología ha proporcionado medios para diseñar sistemas de liberación de fármacos que pueden transportar drogas más efectivamente y mejorar la liberación del fármaco al objetivo elegido. Los sistemas de liberación pueden ser desarrollados utilizando nanoestructuras tales como nanopartículas poliméricas, sistemas lipídicos como liposomas y emulsiones, dendrímeros, nanoestructuras de carbón como nanotubos y micelas auto-ensambladas. Además, nanoestructuras inorgánicas hechas de silicona, nanopartículas metálicas y nanoarmazones, y nanocristales tales como las partículas cuánticas pueden también ser utilizadas. Las ventajas de estos nanosistemas son la disponibilidad de una gran área de superficie y la posibilidad de diseñar nanosistemas multi-funcionales. Por ejemplo, las nanopartículas magnéticas son multi-funcionales en el sentido de que pueden ser utilizadas como sistemas de diagnóstico al igual que como sistemas de liberación de fármacos dirigidos. Una atractiva aproximación para el diagnóstico, la imagen y el tratamiento consiste en funcionalizar la superficie de las nanopartículas magnéticas y dirigirlas a un tejido diana específico con la ayuda de un campo magnético de alto gradiente $\mathrm{y}$, entonces, utilizar un pulso de radiofrecuencia para liberar las drogas contenidas en ellas. Asimismo, las nanopartículas magnéticas pueden ser utilizadas como tratamientos hipotérmicos dirigiéndolos a tejidos cancerosos sensibles al calor y entonces destruir el tejido canceroso mediante la aplicación de un campo magnético AC para calentar las nanopartículas magnéticas (5). Si la terapia génica es una opción, las nanopartículas de ingeniería también pueden ser usadas para liberar genes y SiRNA.

El objetivo de este artículo era exponer al lector a una introducción a la nanotecnología y a cómo varios abordajes basados en la nanotecnología pueden proporcionar mejores métodos de diagnóstico médico y de tratamiento. Adicionalmente, uno debe saber que la respuesta biológica a los nanomateriales de ingeniería y a sus subproductos debe ser cuidadosamente estudiada antes de poner en práctica algunos de los abordajes basados en la nanotecnología discutidos anteriormente. Actualmente, la mayoría de la investigación en este campo se centra en la biocompatibilidad y toxicidad de varios nanomateriales. El objetivo es mejorar los actuales métodos de screening de toxicidad y desarrollar nuevos métodos de examen necesarios para conseguir un uso óptimo de los nanomateriales en las aplicaciones médicas. Uno puede imaginarse muchas aplicaciones potenciales de la nanotecnología a la oftalmología. Finalmente, sería decisivo reunir a grupos de investigación multidisciplinares que integraran su competencia en ciencias físicas y biológicas para hacer avanzar la tecnología médica no limitada sólo a la oftalmología.

\section{BIBLIOGRAFÍA}

1. Tudos AJ, Besselink GJ, Schasfoort RB. Trends in miniaturized total analysis systems for point-of-care testing in clinical chemistry. Lab Chip 2001; 1: 83-95.

2. Figeys D, Lock C, Taylor L, Aebersold R. Microfabricated device coupled with an electrospray ionization quadrupole time-of-flight mass spectrometer: protein identifications based on enhanced-resolution mass spectrometry and tandem mass spectrometry data. Rapid Commun Mass Spectrom 1998; 12: 1435-1444.

3. McGlennen RC. Miniaturization technologies for molecular diagnostics. Clin Chem 2001; 47: 393-402.

4. Smith AM, Dave S, Nie S, True L, Gao X. Multicolor quantum dots for molecular diagnostics of cancer. Expert Rev Mol Diagn 2006; 6: 231-244.

5. Ferrari M. Cancer nanotechnology: opportunities and challenges. Nat Rev Cancer 2005; 5: 161-171. 